\title{
El derecho a la educación del consumidor en Sincelejo*
}

\author{
The right to consumer education in Sincelejo
}

\begin{abstract}
RESUMEN
Los derechos del consumidor se constituyen en la herramienta para evitar el abuso de la posición dominante de una de las partes en la relación de consumo, el proveedor. No obstante, solo será posible tal aspiración en la medida que la sociedad esté educada para asumir un papel protagónico en esta relación.

El siguiente artículo es solo una de las muchas interpretaciones que explican los resultados del trabajo con algunas autoridades y con los representantes de las ligas de usuarios existentes en el municipio de Sincelejo. En él se expone la relación entre la educación de los derechos del consumidor y el papel de estos actores claves en el proceso, explicando cómo finalmente el desconocimiento de las normas, no es un problema meramente informativo sino que transita por el ámbito de las subjetividades del funcionario o representante de las ligas.
\end{abstract}

\section{PALABRAS CLAVE}

Derechos del consumidor, Educación, Sujeto de derechos, Relaciones de consumo.

\section{ABSTRACT}

Consumer rights constitute the tool to prevent abuse of the dominant position of one of the parties in the consumer relationship, the provider. However, such aspiration will only be possible to the extent that society is educated to assume a leading role in this relationship.

The following article is just one of the many interpretations that explain the results of the work with some authorities and with the representatives of the leagues of users existing in the municipality of Sincelejo. It discusses the relationship between the education of consumer rights and the role of these key actors in the process, explaining how finally the ignorance of the rules, is not a merely informative problem but transits through the scope of the subjectivities of the Official or representative of the leagues.

\section{KEYWORDS}

Consumer rights, education, subject of rights, consumer relations.

\section{MARGARITA IRENE JAIMES VELÁSQUEZ}

Abogada, Universidad del Atlántico. Doctorante en Política y Gobierno de la Universidad de Católica de Córdoba, Argentina. Maestra en Educación de los Derechos Humanos del Centro Regional para la Educación de Adultos en América y el Caribe -CREAL-, México. Especialista en DDHH de la ESAP, Colombia. Docente-investigadora del Grupo GICSER. Facultad de Derecho y Ciencias Políticas, Sincelejo, Colombia. margarita.jaimes@cecar.edu.co

YERALDIN ARRIETA RUIZ

Egresada de la Facultad de Derecho y Ciencias Políticas de la Corporación Universitaria del Caribe - CECAR. Joven investigadora del Grupo GICSER. yeraldin.arrieta@cecar.edu.co

* Artículo de investigación de enfoque cualitativo, resultado del proyecto de Investigación en curso "Consuma Caribe", perteneciente al área de Derechos Humanos, desarrollado en el Grupo de Investigación GICSER, financiado por Colciencias y la cofinanciación de la Corporación Universitaria del Caribe en la ciudad de Sincelejo (Colombia). 


\section{INTRODUCCIÓN}

El Estatuto del Consumidor o Ley 1480/2011

nace como una necesidad para proteger al Se resalta que el artículo 78 de la Constituconsumidor o usuario, o sea, aquella perso- ción Nacional de 1991 establece que La ley na natural o jurídica, que como destinatario regulará el control de calidad de bienes y serfinal, adquiera, disfrute o utilice un determi- vicios ofrecidos y prestados a la comunidad nado producto para la satisfacción de una así como la información que debe suminisnecesidad propia, privada, familiar o domés- trarse al público en su comercialización, retica y empresarial cuando no esté ligada in- conociéndole el carácter de derecho humano trínsecamente a su actividad económica*, colectivo. Esto último es importante en tanto consumidor en el Estado Social de Derecho debe ser un elemento esencial y de especial objeto de salvaguarda en la economía del mercado (Henao, 2008)

Por otro lado surge como una necesidad de actualizar la Norma a las nuevas necesidades y realidades a las que se enfrenta el consumidor puesto que el antiguo Decreto 3466 de 1982 que regulaba todo lo relacionado a la idoneidad, la calidad, las garantías, las marcas, las leyendas, las propagandas y la fijación pública de precios de bienes y servicios y dictaba otras disposiciones**, fue concebido para una economía cerrada (Cámara de Representantes, 2010). Su creación anterior a la Constitución de 1991 lo hacía incongruente con la nueva concepción de los

Ley 1480 de 2011. Por medio de la cual se expide el Es Octubre 12 de 2011 . DO № № 48220 .

* Decreto 3466 de 1982. Por medio del cual se dictan normas relativas a la idoneidad, la calidad, las garantías, las marcas, las leyendas, las propagandas y la fijación lidad de sus productores, expendedores y proveedores, y se dictan otras disposiciones. Diciembre 02 de 1982 . tiene el rango de derecho constitucional.

sujetos de derechos y con las tendencias del mercado globalizado.

Se evidencia que el espíritu de la Norma es a defensa de los derechos del consumidor, sin embargo, esto no es posible si el sujeto beneficiario de tales prerrogativas no ha sido educado y debidamente informado sobre e de 2011 establece como derecho la educación del consumidor en cuanto a sus derechos y formas de acceder a ellos pero sin establecer un responsable directo. Es de aclarar que este derecho es distinto al descrito en el artículo 59 del Estatuto que reza: “En desarrollo de las funciones que le han sido asignadas a la Superintendencia de Industria formar y capacitar en materia de protección al consumidor..." mientras que el primero busca construir al sujeto de derechos que está ligado al concepto del ejercicio de la ciuligado a la relación clientelar y de consumo.

Teniendo en cuenta que el Estatuto entró en vigencia en el año 2012, es de suma importancia discutir si las acciones que realiza contenido y el alcance de estos. La Ley 148 y Comercio esta propenderá por difundir, indadanía, mientras que el segundo está más institucionalidad pública, las universidades y las ligas de consumidores en la ciudad de Sincelejo materializan el derecho a la educación del consumidor.

Para ello, se examinarán los resultados de grupo focal con autoridades en la materia, realizado en el marco del Proyecto de Investigación "Fortalecimiento local de los derechos de los consumidores: promoción y protección de los derechos del consumidor, en a ciudad de Sincelejo, en el marco del nuevo Estatuto del Consumidor, periodo 2015 2016" que está integrado al "Programa de fortalecimiento de las capacidades regionales y locales de los consumidores: promoción del marco jurídico de las competencias establecidas en el nuevo Estatuto del Consumidor (alcaldes, asociaciones de consumidores y consultorios jurídicos en Santa Marta, Cartagena y Sincelejo) periodo 2015-2016", que tiene como objetivo: "Promover el fortalecimiento de los derechos de los consumidores en tres ciudades de la costa Caribe colombiana (Cartagena, Santa Marta y Sincelejo), partir de la educación, protección, garantía y defensa en el ejercicio de sus derechos económicos en el marco de las prerrogativas establecidas en el nuevo Estatuto del Consumidor en el periodo 2015-2016".

\section{El sujeto de derechos}

sujeto de derechos, según Abraham Magendzo (s.f.) es aquella persona que conoce sus derechos y además sabe ejercerlos porque se reconoce con el poder para exigirlos. El sujeto de derechos es crítico de las realida- des que le circundan y participa activamente en la transformación de aquello que le impide realizarse plenamente como persona.

En términos jurídicos el sujeto de derechos es aquella persona que actúa en una relación jurídica, es decir, aquella dotada de capacidad jurídica. En palabras de Hans Kelsen (1960, p.101)

El concepto jurídico de sujeto de derecho expresa solamente la unidad de una pluralidad de deberes, de responsabilidades y de derechos subjetivos, es decir, la unidad de una pluralidad de normas que determinan estos deberes, responsabilidades $y$ derechos subjetivos.

En síntesis, el sujeto de derecho es aquella persona natural dotada de capacidad para asumir sus derechos y responder por sus deberes. Hablamos de la humanidad del sujeto en tanto su capacidad de análisis y poder transformador.

\section{La educación}

La educación es pilar fundamental en el desarrollo humano, su valía radica en el poder transformador y socializador de nuevas realidades entre congéneres. Al respecto Freire (1971) señalaba que solo la educación posibilita la construcción de la sociedad humana, en tanto ella es praxis, reflexión y acción del hombre sobre el mundo para transformarlo en una relación dialogante con los otros. Es este no silencio una apuesta política para la liberación. 
En la Afirmación de Amman la comunidad internacional reconoce que la educación es transversal a todos los ámbitos de la vida hu-

mana y reafirma su poder transformador al sostener que

La educación da poder. Es la clave para establecer y fortalecer la democracia y el desarrollo, la cual es tanto sustentable como humana y basada en la paz hacia un respeto mutuo y justicia social. Además, $n$ un mundo en donde la creativid conocimiento juegan un rol importante, $\mathrm{e}$ derecho a la educación no es nada menos que el derecho a participar en el mundo moderno

En el campo de los derechos humanos se ha instituido como derecho en el artículo 26 de la Declaración Universal de Derechos Humanos estableciendo que su objeto es el desarrollo de la persona humana, en aras de garantizar la tolerancia y las relaciones de amistad entre las naciones. La Observación No. 13 del Comité de Derechos Económicos, Sociales y Culturales de las Naciones Unidas amplió su contenido al afirmar que:

a educación es un derecho humano intrínseco y un medio indispensable de realizar otros derechos humanos. Como derecho del ámbito de la autonomía de la persona, la educación es el principal medio que permite a adultos y menores, marginados económica y socialmente, salir de la pobreza y participar plenamente en sus comunidades. (1999, parr. 1)

Más adelante se vincularía con la noción de desarrollo y medioambiente sustentable al exponer que la educación

(...) deberá ser reconocida como un proceso por medio del cual los seres humanos $y$ las sociedades pueden alcanzar su entero potencial. La educación... promueve el de recho sustentable y mejora la capacidad de las personas para manejar temas como medio ambiente y desarrollo... (Agenda 21, capítulo 36 párrafo 3 Cumbre Mundia de la Tierra realizada en Río)**

Ya en el orden jurídico interno, la Corte Constitucional colombiana ha dicho respecto a derecho a la educación que

[i]ndudable que el derecho a la educación pertenece a la categoría de los derechos fundamentales, pues, su núcleo esencial, comporta un factor de desarrollo individual y social con cuyo ejercicio se materializa el desarrollo pleno del ser humano en todas sus potencialidades. Esta Corporación, también ha estimado que este derecho constituye un medio para que el individuo se integre efectiva y eficarmente a la sociedad; de allí su especial categoría que lo hace parte de los derechos esenciales de las personas en la medida en que

* Naciones Unidas, Cumbre Mundial de la Tierra, Programa 21 (Programa de Acción de las Naciones Unidas e
Rio), 1998, cap. 36, parrr. 3; cap. 3, pár. 2, y cap. 24, pár. el conocimiento es inherente a la naturaleza humana. La educación está implícita como una de las esferas de la cultura y es el medio para obtener el conocimiento $y$ lograr el desarrollo y perfeccionamiento del hombre. La educación, además, realiza el valor y principio material de la igualdad que se encuentra consignado en el Preámbulo y en los artículos 5, 13, 68 y 69 de la C.P. En este orden de ideas, en la medida en que la persona tenga igualdad de probabilidades educativas, tendrá igualdad de oportunidades en la vida para efecto de realizarse como persona". (Sentencia T-087 de 2010)

De lo anterior se infiere que la educación es un medio y un fin en sí misma. Es un medio para superar la pobreza y la desigualdad en tanto facilita el desarrollo de las capacidades y es un fin en tanto está dotada de un contenido jurídico que le otorga el reconocimiento de derecho humano.

\section{Las relaciones de consumo}

En una sociedad globalizada las relaciones sociales y las relaciones económicas de mercado están estrechamente ligadas a las relaciones de consumo. Todas las necesidades básicas se satisfacen a través del consumo de bienes y servicios ofertados en el mercado.

Ahora bien, las relaciones de consumo no se centran meramente en el acto d prar, consumir o utilizar servicios, sino que compromete otro tipo de actos que perm ten equilibrar las relaciones de poder que existen entre el productor y el consumidor. Esos actos implican analizar, por ejemplo los procesos de elaboración y distribución de los productos, aprender a usar los bienes y servicios que se consumen, ser capaces de analizar críticamente las estrategias publicitarias de los productos o ejercitar los mecanismos de protección establecidos en el ordenamiento jurídico cuando un derecho ha sido vulnerado.

De acuerdo al Estatuto del Consumidor, en Colombia el derecho a la educación del consumidor tiene una doble connotación, por un lado es una relación derecho/obligación que impone cargas al Estado y a los productores en beneficio de los consumidores, y por el otro, es un servicio susceptible de consumo.

\section{Educación del consumidor o educación para} el consumo

Al hablar tanto de educación como de consumidor, estamos hablando de un fenómeno global de consumo (Castillejo et al., 2013), que se presenta en el diario vivir de todas las sociedades del mundo. Es por lo que se hace necesario educar para alcanzar un consumo responsable por cada uno de los seres humanos, no solo en los adultos que son en gran parte los que poseen los recursos económicos, sino también los niños, niñas y adolescentes que juegan un papel importante en la conducta consumista de todos los países del mundo.

En cuanto a la educación para el consumo, 
Castillejo et al. (2013) afirmaron que esta do racionales a la hora de adquirir y disfrutar se logra, ante todo, con el ejemplo, con la de productos, bienes y servicios que ofrece vivencia directa en un ambiente de modera- el mercado. En otras palabras, la educación

ción y responsabilidad respecto a los bienes para el consumo introduce racionalidad y y servicios consumidos y los padres son los crea personas responsables. primeros modelos a imitar, los que determinan en mayor medida un futuro consumo responsable o irresponsable, dejando claro por otro lado, que la escuela, por sí sola, no podrá lograr una educación para el consumo eficaz si no establece una auténtica acción coordinada con las familias, porque de ellas proceden la socialización de los modos de conducta que hacen posible el consumo.

Trímboli (2004) afirma que la calidad de consumidores es algo que el ser humano jamás puede evitar, debido a las constantes relaciones de consumo que se manejan en el diario vivir de la sociedad actual, pero sí pueden optar por un comportamiento adecuado que permita vivir en colectividad sin afectar los derechos que las demás personas tienen, de empoderarse de los derechos y correlativos deberes para defender eventuales vulneraciones, eligiendo la forma de consumir responsablemente. Elección adecuada que se va a alcanzar en la medida que las personas se encuentren educadas.

Para concluir con la posición de estos autores, la educación para el consumo debe tener su génesis desde el seno mismo de la familia apostándole tal forma a la enseñanza desde la infancia, permitiendo así poder garantizar a futuro, adultos responsables en la toma de decisiones, ajustadas a las necesidades, sien-
A nivel mundial las organizaciones de consumidores promueven iniciativas para educar jóvenes y adultos. En el continente asiático, por ejemplo, incluyen temas como satisfacción de necesidades básicas, acción de corporaciones transnacionales, productos y medicamentos peligrosos, entre otros. En Europa se encuentra la Escuela Europea de Consumidores, en América Latina y el Caribe, los países pioneros en las iniciativas de educación al consumidor lo lideran: México, Brasil, Argentina y Jamaica.

\section{MÉTODO}

\section{Tipo de estudio}

Este estudio de corte sociojurídico pretendio 1480 de 2011 en materia de educación de consumidor desde las condiciones situacionales que permiten la materialización del fin perseguido, por ello se trabajó desde el en foque cualitativo que analiza los escenarios las personas integralmente desde una log de interpretación y no como variables (Taylor \& Bogdan, 1987). Para ello se aplicaron diversas herramientas de las Ciencias Sociales, pero para efectos de este escrito los resultados descritos serán los arrojados en las trevistas grupales. determinar el significado y alcance de la Ley

\section{Participantes}

RESULTADOS

Nuestra población universo fueron los y las funcionarias de entidades públicas y privadas que de acuerdo a la Ley 1480 de 2011 y otras normas del ordenamiento jurídico, tienen estipuladas las acciones de promoción y protección de los derechos de los consumidores en el municipio de Sincelejo.

Así pues, en cuanto a la institucionalidad pública se convocó a personal de la Alcaldía Municipal, de la Personería Municipal, la Defensoría del Pueblo y a los delegados de la Superintendencia de Servicios Públicos Domiciliarios. En cuanto a organizaciones e instituciones privadas se invitó a los representantes de las ligas y asociaciones de consumidores en el municipio y al Consultorio Jurídico de CECAR por ser el único activo en el año 2015.

Del total de veinte personas convocadas solo asistieron once, con quienes se trabajó en tres sesiones.

Para la consecución de datos se acudió a la técnica de entrevista grupal que se caracteriza por la búsqueda de significado entre múltiples análisis. El grupo de personas convocadas a los grupos focales discutieron desde la experiencia personal lo concerniente a los derechos del consumidor y su rol en la materialización de ellos.

La fase de análisis se realizó mediante la codificación de la información y la interpretación de los hallazgos.
En cuanto al conocimiento de la norma espeE aquellas relacionadas con la prestación de un servicio público se encontró:

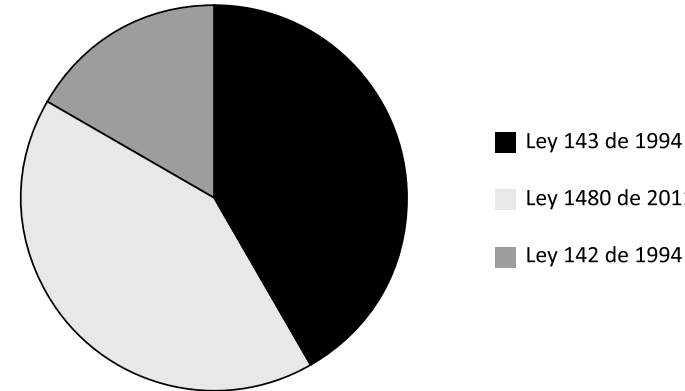
Figura 1.

Se observa que el $45,4 \%$ de los funcionarios y líderes conoce la Ley 143 de 1994, el mismo porcentaje conoce la Ley 1480 de 2011 y el restante conoce la Ley de Servicios Públicos. Afirmaron conocer las leyes los representantes de la Superintendencia de Servicios Públicos, del Consultorio Jurídico de CECAR, de la Asociación Departamental de Usuarios de Servicios Públicos y los representantes de la Liga de Consumidores. En suma, solo cinco conocen las normas, el resto $(54,6 \%)$ desconoce todas las normas que protegen las relas de consumo.

De los cinco que identificaron la Ley 1480 de 2011, tres son representantes de la Liga de Usuarios de Consumidores, uno de la Asociación Departamental de Usuarios de Servicios Públicos y uno del Consultorio Jurídico de CECAR, y dos de ellos mismos (un representante de Consultorio Jurídico y otro de la Asociación Departamental de Usuarios de Servicios Públicos), identificaron además la Ley 142 de 1994. 
Se encontró que dentro de los desconoce- han apuntado a resolver aspectos relativos dores de la norma se encontraban algunos a la eficacia de las normas que protegen representantes de la Asociación Departa- los derechos de los consumidores desde la mental de Usuarios de Servicios Públicos, la mirada del cumplimiento de los estándare Personería Departamental y la Asociación de internacionales o desde la óptica del DereUsuarios de Coomeva.

\section{DISCUSIÓN}

La Ley 1480 de 2011 expresa que la educación para el consumo y como consumidores es un principio general y un derecho de la ciudadanía; no obstante, no se asignaron responsabilidades claras y expresas a ningún organismo público o privado, lo que tiene repercusiones negativas y directas en el cumplimiento del objetivo principal del Estatuto del Consumidor

En ocasiones suele confundirse el derecho a la información con el derecho a la educación del consumidor. Si bien estos dos derechos se complementan, difieren en cuanto a su contenido, su alcance y en los sujetos responsables de su cumplimiento. Mientras que el derecho a la educación constituye un proceso encaminado a formar sujetos de derechos capaces de ser ejercer y proponer en el marco de las relaciones de consumo la información busca garantizar que el consumidor conozca las propiedades del producto que está comprando, o bien que está adquiriendo. Incluso la misma Ley lo reconoce como deber en cabeza del consumidor.

Algunos estudios sobre los derechos del consumidor que se han realizado en Colombia internacionales o desde la óptica del Derecho Comparado y no desde el derecho a la educación del consumidor, que como se h dicho anteriormente debe propender por garantizar la materialización de los contenidos normativos desde la condición del sujeto de derechos.

Esto adquiere relevancia cuando se observa que el $54,6 \%$ de los funcionarios públicos y privados desconocen la normatividad relativa a las relaciones de consumo, lo que afecta gravemente la capacidad de estos para promover, divulgar y educar a la ciudadanía en el ejercicio de los derechos como consumidores. Esto explica las razones por las que no existen programas encaminados a educar la ciudadanía en la cultura del consumo responsable y el ejercicio de sus derechos.

Se puede inferir además que estos funcionarios y representantes de organizaciones no se reconocen como usuarios o consumidore y de allí su desinterés en formarse para cumplir a cabalidad la misión encomendada por la sociedad. En el caso de los representantes la ley, existe una relación directa entre el conocimiento de la norma y el autorreconocimiento en una relación de consumo, ya que lo primero conlleva a lo segundo. No es retórico el comentario, pues, cuando un funcionario o representante siente que compar- está habilitado para decantar hasta lo sumo los contenidos normativos.

Adecuando lo dicho por Magendzo (2013) solo el sujeto de derecho está en capacidad de cuestionar el entorno y proponer transformaciones desde el lugar que ocupa en sociedad. Con esto queremos decir que el proceso de educación para el consumo traspasa los contenidos normativos para adentrarse en la esfera de las subjetividades de funcionario o representante encargado de promover la educación para el consumo.

La inexistencia de programas reales de educación para el consumo, explica además e por qué líderes y funcionarios se convierten en operadores logísticos de la norma y no en verdaderos defensores y protectores de los derechos de los consumidores. Aplicando la analogía de que un ciego no puede guiar a otro ciego, nos encontramos ante un obstáculo, que si bien no es insuperable, requiere de ingentes esfuerzos para lograrlo. Ciertamente, compete al Estado establecer los modos y proveer los medios para garantizar la educación formal y no formal del derecho al consumo, pero desde una mirada del consumo responsable.

Como bien se dijo adelante, la educación se constituye en el medio idóneo para eliminar la discriminación y fomentar las relaciones de igualdad, máxime en relaciones en las que una de las partes, el proveedor en este caso, ostenta el poder económico frente a la necesidad de satisfacción de necesidades básicas, reales o imaginarias de los consumidores.

\section{CONCLUSION}

La educación del consumidor es una necesidad que requiere acciones urgentes del Estado colombiano en aras de garantizar que en as relaciones de consumo no se abuse de posición dominante del productor o proveedor. Para ello, debe establecerse claramente as responsabilidades a fin de otorgarle poder de exigibilidad.

Es importante que los funcionarios y representantes de las ligas de protección de usuarios conozcan todo el andamiaje jurídico que soporta los derechos del consumidor en todas sus categorías y diversidad de rutas jurídicas y administrativas.

Igualmente es imprescindible que surja el factor subjetivo, es decir, la empatía entre pares para que quienes han asumido voluntariamente o por mandato misional la tarea de proteger los derechos de los consumidores, puedan comprender más allá de la norma los matices que trae consigo el ejercicio de los derechos del consumidor; entre ellos, la necesidad imperiosa de educar a la sociedad sobre el consumo responsable y los derechos que ello conlleva. 


\section{REFERENCIAS BIBLIOGRÁFICAS}

Cámara de Representantes (09 de septiembre de 2010). Congreso Visible.org. Obtenido de Gaceta del Congreso No. 626, Proyecto de Ley 089 de 2010: http://www.imprenta.gov. co/gacetap/gaceta.indice?v_num $=626 \& v_{\text {_ }}$ anog $=2010$

Castillejo, J. L., Colóm, A. J., Pérez-Geta, P. Mā A., Neira Rodríguez, T., Sarramona, J., Touriñán, J. M. \& Vázquez, G. (2013). Educación para el consumo. Educación, XX1, 14(1), 35-58.

Comité de Derechos Económicos, Sociales y Culturales de las Naciones Unidas (1999). Aplicación del Pacto Internacional de los Derechos Económicos, Sociales y Culturales. En Observación general 13, El derecho a la educación (artículo 13 del Pacto), (21 periodo de sesiones, 1999), U.N. Doc. E/C.12/1999/10.

Constitución Política de Colombia [Const.] (Julio 7 de 1991). Colombia.

Corte Constitucional. Sentencia T-087 de 2010 (M.P. Humberto Antonio Sierra Porto: Febrero 11 de 2010).

Decreto 3466 de 1982. Por medio del cual se dictan normas relativas a la idoneidad, la calidad, las garantías, las marcas, las leyendas, las propagandas y la fijación pública de precios de bienes y servicios, la responsabilidad de sus productores, expendedores y proveedores, y se dictan otras disposiciones. Diciembre 02 de 1982.

Freire, P. (1971). La educación como práctica de la libertad. 3a ed. Montevideo, Uruguay: Edit. Tierra Nueva.
Gaceta del Congreso No. 352 (01 de junio de 2011). Informe de Ponencia para Primer debate al Proyecto de Ley 252 de 2011 Senado, 089 de 2010 Cámara. Obtenido de Ley 1480 de 2011 Artículo 59. Facultades Administrativas: http://www.imprenta.gov.co/gacetap/ gaceta.mostrar_documento?p_tipo $=22 \&$ _ numero $=252 \&$ p_consec $=29261$

Henao, M. C. (2008). Libertad de empresa en el Estado Social de Derecho. Bogotá: Universidad Externado de Colombia.

Kelsen, H. (1960). Teoría pura del Derecho. 1ạ ed. Buenos Aires: Edit. Eudeba.

Ley 1480 de 2011. Por medio de la cual se expide el Estatuto del Consumidor y se dictan otras disposiciones. Octubre 12 de 2011. DO: 48220.

Magendzo, A. (2013). La educación en derechos humanos: diseño problematizador. Recuperado de: http://www.corteidh.or.cr/ tablas/r24460.pdf

Naciones Unidas, Cumbre Mundial de la Tierra, Programa 21 (Programa de Acción de las Naciones Unidas en Río), 1998.

Naciones Unidas, Foro Consultivo Internacional sobre Educación para Todos. Afirmación de Amman, 1996.

Taylor, S. J. \& Bogdan, R. (1987). Introducción a los métodos cualitativos de investigación. Barcelona: Edit. Paidós.

Trímboli, J. (2004). Educación del consumidor. Realidad y perspectivas. Consumer's International. Recuperado de: http://consumidoresint.org 